\title{
Rumination Syndrome: Unknown Pathology Easy to Diagnose With High-resolution Impedance Manometry
}

TO THE EDITOR: We present the case of a 63-year-old patient, with a long history of recurrent regurgitation of undigested food that occurred about 15 minutes after eating and could last for hours. Vomiting was absent. She complained of occasional heartburn but repeated trials of proton pump inhibitors and prokinetics failed to relieve symptoms.

Previously, an extensive investigation including an upper endoscopy and blood test were rigorously normal. To exclude gastroesophageal reflux disease, her physician ordered a 24-hour impedance $\mathrm{pH}$ monitoring that we completed in our motility unit. Remarkably, this showed a multitude of episodes that the software identifies as non-acid reflux after meals without any episodes during the nocturnal period. It was observed that immediately after a normal swallow these episodes appeared with a retrograde decrease in the impedance trace that reached the proximal sensor followed by anterograde recovery, with the esophageal $\mathrm{pH}$ above 4 during these events. Accordingly, high-resolution impedance manometry (HRIM) was performed in the supine position, performed as follows: (1) standard protocol study with 10 liquid swallows of $10 \mathrm{~mL}$ of saline that was normal and (2) solid meal consisting of chicken sandwich which revealed a sudden increase in intragastric pressure ( $>30 \mathrm{mmHg}$ ) that reached and opened the upper esophageal sphincter. The impedance showed that the increase of the gastric pressure caused a retrograde movement of the intragastric content to the hypopharyngeal area followed by a swallow that clarifies the bolus (Fig. 1), occurring a number of times after the ingestion (Fig. 2).

Consequently, we concluded the diagnosis of rumination syndrome, proposing biofeedback treatment.

We present an example of a pathology whose diagnostic ap-
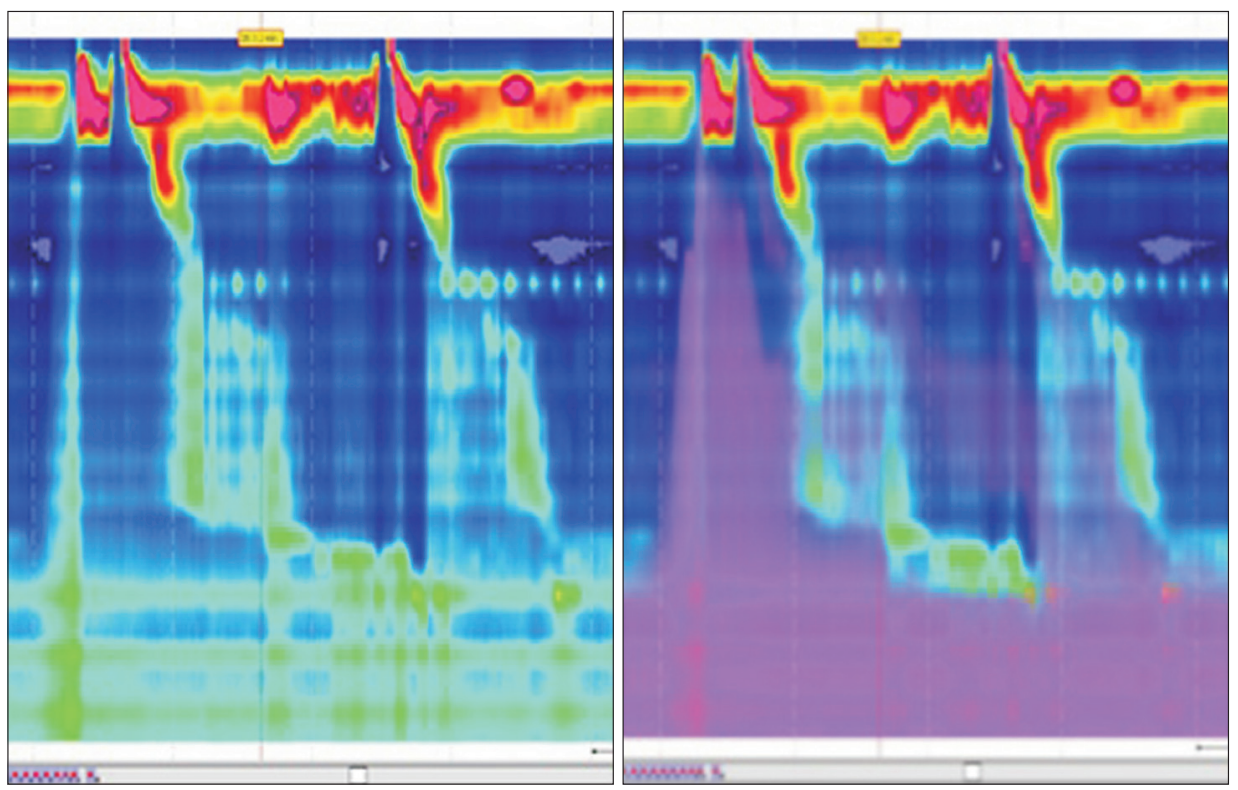

Figure 1. High-resolution impedance manometry. With the solid meal a sudden increase in intragastric pressure $(>$ $30 \mathrm{mmHg}$ ) that reached and opened the upper esophageal sphincter was observed (left image). The impedance revealed that the increase of the gastric pressure caused a retrograde movement of the intragastric content to the hypopharyngeal area followed by a swallow that clarifies the bolus (right image). 


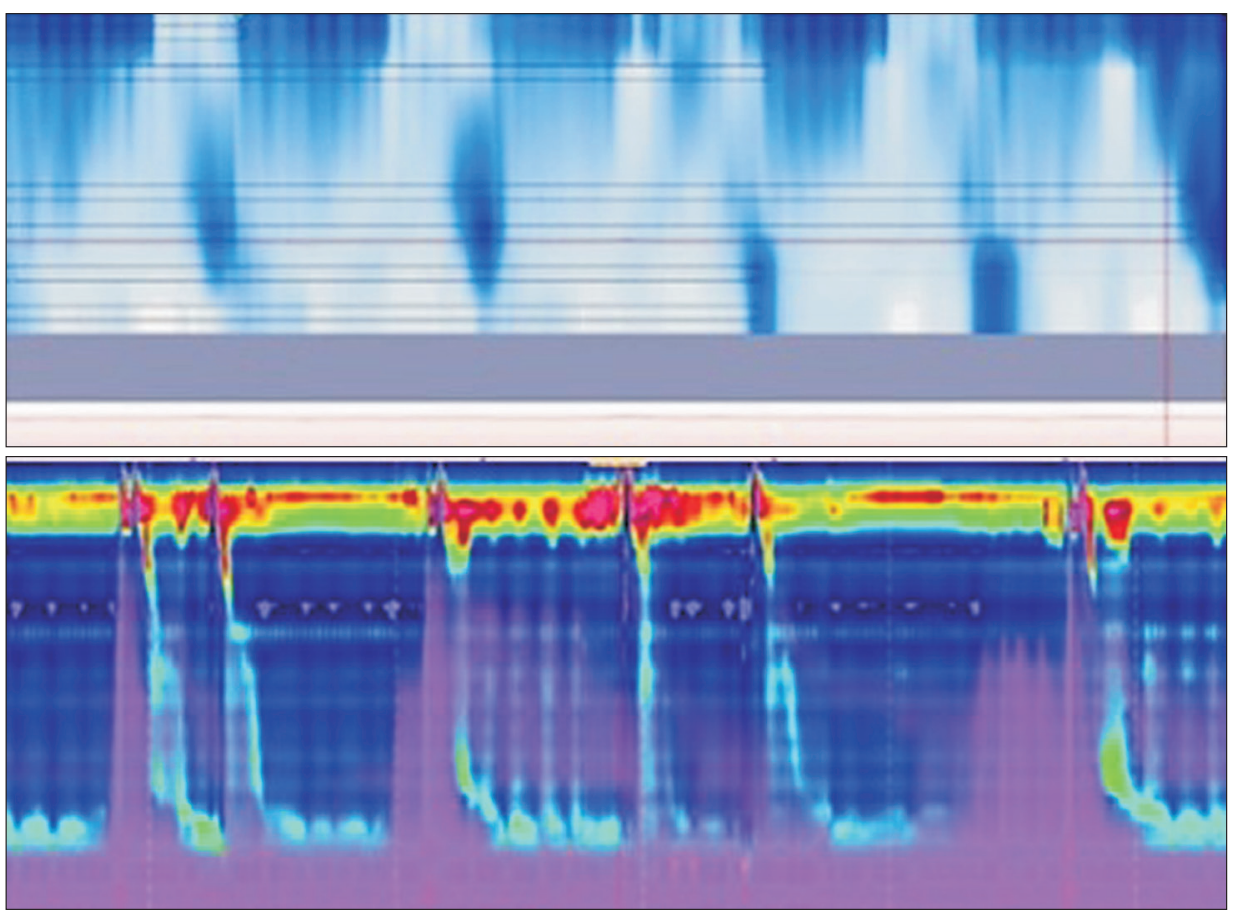

Figure 2. Comparative image of the episodes of rumination objectified by 24 hour impedance $\mathrm{pH}$ monitoring (upper image) and high-resolution impedance manometry (lower image), which occur several times after ingestion. proach is not easy in many cases due to the limited knowledge of this condition among attending professionals in terms of the clinical presentation, diagnosis, and treatment. ${ }^{1}$ Patients with rumination syndrome are often misdiagnosed and undergo numerous avoidable tests.

These episodes are typically induced by a rise in intragastric pressure, generated by a voluntary contraction of the abdominal wall and intercostal musculature which causes relaxation of the lower esophageal sphincter, but the overall process of rumination is generally involuntary, non-intentional. ${ }^{2,3}$

The diagnosis of rumination syndrome in adults is based on the Rome IV criterion. ${ }^{4}$ A careful clinical history is very important in the diagnosis and in many cases is enough to establish it. In addition, this disorder needs to be distinguished from other gastrointestinal diseases that also have regurgitation/vomiting as the main symptom, including gastroesophageal reflux disease, achalasia, gastroparesis, and cyclic vomiting syndrome. ${ }^{5,6}$ However, in cases where there are diagnostic doubts, HRM and HRIM with a solid meal allow us to clarify the diagnosis. As we have seen in our case, the $\mathrm{pH}$-impedance monitoring is often not able to distinguish between the episodes of rumination and reflux, nevertheless in rumination, in the majority of episodes, the refluxed material reaches the proximal esophagus. ${ }^{7,8}$

Pharmacological treatment is not very useful in rumination syndrome. ${ }^{9}$ Proton pump inhibitors and prokinetics are both inef- fective. The usefulness of baclofen in this context has recently been demonstrated. ${ }^{10}$ However, the treatment that nowadays appears to obtained better short-term results is aimed at modifying the final mechanism that produces it by re-education of abdominal contractions and diaphragmatic breathing with biofeedback. ${ }^{5}$ Funduplication has been proposed but with few results and even with complications such as rupture of the funduplication by excess intragastric pressure. More multicenter, prospective long-term studies are needed to evaluate long-term outcomes.

\section{Esperanza Martos Vizcaino, ${ }^{1}$ Fernando Canga Rodriguez-Valcárcel, and Constanza Ciriza de Los Ríos}

${ }^{1}$ Departament of Gastroenterology, Digestive Motility Unit, Hospital Universitario Gregorio Marañón, Madrid, Spain; and ${ }^{2}$ Departament of Gastroenterology, Digestive Motility Unit, Hospital Universitario 12 de Octubre, Madrid, Spain

1. Giménez Casado A, López Liñán MJ, Barba Orozco E, et al. [Rumination syndrome: diagnostic and therapeutic difficulties of a not so uncommon disorder.] An Pediatr (Barc) 2017;88:100-105. [Spanish]

2. Hejazi RA, McCallum RW. Rumination syndrome: a review of current concepts and treatments. Ame J Med 2014;348:324-329.

3. Kessing BF, Smout AJ, Bredenoord AJ. Current diagnosis and management of the rumination syndrome. J Clin Gastroenterology 2014;48:478483

4. Barba E, Burri E, Accarino A, et al. Biofeedback-guided control of 
abdominothoracic muscular activity reduces regurgitation episodes in patients with rumination. Clin Gastroenterol Hepatol 2015;13:100-106, e1.

5. Tucker E, Knowles K, Wright J, Fox MR. Rumination variations: aetiology and classification of abnormal behavioural responses to digestive symptoms based on high-resolutionmanometry studies. Aliment Pharmacol Ther 2013;37:263-274.

6. Kessing BF, Govaert F, Masclee AA, Conchillo JM. Impedance measurements and high-resolution manometry help to better define rumination episodes. Scan J Gastroenterol 2011;46:1310-1315.

7. Kessing BF, Bredenoord AJ, Smout AJ. Objective manometric criteria for the rumination syndrome. Am J Gastroenterol 2014;109:52-59.

8. Singendonk MMJ, Oors JM, Bredenoord AJ, et al. Objectively diagnosing rumination syndrome in children using esophageal $\mathrm{pH}$-impedance and manometry. Neurogastroenterol Motil 2017;29:e12996.

9. Saleh CM, Bredenoord AJ. Utilization of esophageal function testing for the diagnosis of the rumination syndrome and belching disorders. Gastrointest Endosc Clin N Am 2014;24:633-642.

10. Blondeau K, Boecxstaens V, Rommel N, et al. Baclofen improves symptoms and reduces postprandial flow events in patients with rumination and supragastric belching. Clin Gastroenterol Hepatol 2012;10:379-384.

\section{Financial support: None.}

Conflicts of interest: None.

Author contributions: All authors were involved in the clinical management of the patient, all planned the communication of the case, and all were involved in the design of the report. Esperanza Martos Vizcaino drafted the manuscript; and Constanza Ciriza de Los Ríos and Fernando Canga Rodriguez-Valcárcel review and made contributions for the manuscript and approved the final draft submitted. 